three-pronged 22G FNB needle introduced from July to December. Standardised tissue sampling technique was undertaken with 3 samples obtained, each with 5 passes made using a fanning technique. Samples were placed directly into cytorich red fixative. Patients were excluded if the needle size was not recorded, alternate size needle used, or biopsy protocol not followed. Accuracy was calculated and compared between those with and without SEMS and between FNA and FNB procedures. Patients had a confirmed benign diagnosis if there was no progression or an improvement in mass appearance after at least 3 months of follow up.

Results After exclusions, 128 patients undergoing 145 pancreatic biopsies were recruited (10 patients had 2 procedures and 4 patients had 3 procedures). A final diagnosis of malignancy was made in $93.8 \%(136 / 145)$. Overall accuracy of pancreatic biopsy was $84.1 \%(122 / 145)$. FNB accuracy was significantly higher compared to FNA (89.9\% [80/89] versus $75.0 \%$ [42/ 56], $\mathrm{p}=0.02$, Odds Ratio [OR] 2.96, 95\% confidence interval [CI] 1.18-7.41). The overall accuracy in the presence of SEMS was $82.5 \%(52 / 63)$ which was not significantly different than those without SEMS 85.4\% (70/82) (OR 1.23 95\% CI 0.50-3.01 $\mathrm{p}=0.64)$. The accuracy of sampling pancreatic solid lesions when a SEMS was present with FNB () was not significantly higher compared to FNA $(82.9 \%$ [34/41] versus $81.8 \%$ [18/22], p=0.91, OR 0.92 95\% CI 0.24-3.60).

Conclusions The accuracy of EUS-FNA for pancreatic solid lesions exceeds the current recommendations and is increased with use of FNB. National standards should take improved accuracy with FNB into account when being updated. In our experience use of FNB does not improve the accuracy of pancreatic tissue sampling in the presence of a SEMS.

\section{OWE-33 CAN MODERATE ALCOHOL CONSUMPTION DECREASE PANCREATIC DUCTAL ADENOCARCINOMA (PDAC) RISK? A SYSTEMATIC REVIEW AND META-ANALYSIS}

${ }^{1}$ Thomas Payne*, 1,2Stephen Lam, 1,2 Andrew Hart. 'Norwich Medical School, Norwich, UK; ${ }^{2}$ Norfolk and Norwich University Hospital, Norwich, UK

\subsection{6/gutjpl-2019-BSGAbstracts.293}

Introduction PDAC is increasing in incidence and has the worst survival of any malignancy. Lifestyle choices to reduce risk, based on robust evidence, are required. There are plausible biological mechanisms for how a moderate intake of alcohol (UK recommendations of $\leq 14$ units/week) could prevent PDAC. These are alcohol: mitigating against pathological effects of KRAS genetic mutations, decreasing excess mitogenic insulin secretion, and the effects of antioxidants in alcoholic drinks. These mechanisms must be supported by robust epidemiological data to infer causality.

The aim of this work is to systematically review prospective cohort studies, selected according to predefined criteria, to assess if a moderate alcohol consumption is inversely associated with PDAC risk. Cohort studies minimise biases inherent in other observational methodologies.

Methods Medline, PubMed and EMBASE electronic databases were searched for prospective cohort studies investigating a moderate alcohol consumption and PDAC up to January 2019. The inclusion criteria were: $>100$ PDAC cases, definition of moderate consumption within UK guidelines, clear description of the comparator group, reporting effect sizes as risk/hazard ratios (RR/HR) and adjustment for covariates including: age, sex, smoking, body mass index and diabetes mellitus. Pooled effect sizes were calculated using an inverse variance random-effects model.

Results Four prospective cohort studies meeting inclusion criteria were identified $(9,090$ PDAC cases, $57.5 \%$ female, mean age at diagnosis was 61.1 years). The cohorts were in the Netherlands, UK, pan-European and the US. The pooled effect size showed no association between a moderate alcohol consumption and PDAC risk $\left(\mathrm{HR}=0.97,95 \% \mathrm{CI}\right.$; 0.91-1.03, $\mathrm{I}^{2}$ for heterogeneity $0.0 \%$ ). There was no evidence of publication bias (Egger's $p=0.404$ ). Three cohorts investigated moderate intake of different beverages, reporting no association with either: wine $\left(\mathrm{HR}=0.95,95 \% \mathrm{CI} ; 0.83-1.09, \mathrm{I}^{2}=0.0 \%\right)$ beer $\left(\mathrm{HR}=0.96,95 \% \mathrm{CI} ; 0.79-1.17, \mathrm{I}^{2}=0.0 \%\right)$ or liquor $\left(\mathrm{HR}=1.18,95 \% \mathrm{CI}, 0.89-1.57, \mathrm{I}^{2}=71.7 \%\right)$. There was no biological gradient within the moderate range.

Conclusions The plausible biological mechanisms for a protective effect of alcohol are not supported by the epidemiological work. Drinking alcohol moderately cannot be recommended to decrease PDAC risk in the general population. Further research in a large prospective cohort which investigates risk in specific groups such as smokers and according to body mass index is required to see if alcohol may be beneficial in high risk groups for PDAC.

\section{Posters}

\section{PWE-063 SINGLE SESSION EUS-ERCP; AN EFFECTIVE APPROACH}

Umair Kamran*, Ratul Adhikary, Damien Durkin, Robert Glass, Srisha Hebbar. University Hospitals of North midlands, Stoke on trent, UK

\subsection{6/gutjnl-2019-BSGAbstracts.294}

Introduction The primary objective of this study was to look at the safety and efficacy of single session endoscopic ultrasound (EUS) and endoscopic retrograde cholangiopancreatography (ERCP) procedures at a tertiary care hospital.

Methods We retrospectively reviewed patient data from a prospectively maintained EUS-ERCP database from October 2017 to January 2019. Descriptive statistics were performed on Microsoft excel. We evaluated the reasons for the planned combined procedures, procedural characteristics and outcomes. Results 55 patients were included in this study. Majority were females (35 vs 20) and median age was 69 years. 58\% of patients (32) were referred for ERCP only but requests were reviewed by the consultant with expertise in performing both procedures and vetted for combined procedures instead. Most common reason for booking patients on combined slot was inability to identify cause of biliary obstruction (58\%) on prior imaging followed by small $(<5 \mathrm{~mm})$ non-obstructing stone in asymptomatic patients $(15 \%)$ and fine needle aspiration/biliary drainage (15\%). 25 (45\%) patients did not require ERCP as no pathology was identified on EUS. Average total time required for the combined procedure was 30 minutes (range: 20-50 minutes). Two patients had propofol and the median dose of fentanyl and midazolam used in the remaining patients were $100 \mathrm{mcg}(50-200 \mathrm{mcg})$ and $4 \mathrm{mg}(2-5 \mathrm{mg})$ respectively. One serious complication of ERCP was experienced.

Although the patients who were symptomatic on the day of the procedure were more likely to require ERCP following 